\title{
New Ways of Learning through the Global Information Infrastructure
}

\author{
Jacob Slonim $^{a}$ and Michael Bauer ${ }^{b}$
}

\begin{abstract}
With increased access to computer-based information sources and the growth of national information infrastructures, society is shifting to one that is informationbased. The result of this shift is new types of workers and new applications; both will become the driving forces behind the evolution of systems, particularly open systems. What is required, then, is a broader view of an "open system".

In this paper, we first examine some of the technological and social transitions underway as society becomes more information-based. We then look at a particular emerging application area, life-long learning, and its technological and social-economic requirements. We broaden the notion of an open system and discuss the challenges faced by developers of open systems prompted by the application demands.
\end{abstract}

Keyword Codes: C.2.4., H.4.3, K.3.1

Keywords: open systems, distributed applications, information infrastructure, life-long learning

\section{INTRODUCTION}

The growing accessibility of computing resources, communication infrastructures and digitized information is causing a major shift in society's behaviour. As computer-based information sources become more accessible and as the need for timely information in many different organizations grows, our technical-based society is shifting to become an information-based one. In looking at this shift from a broad perspective, one can see three fundamental dimensions. First, there is technology: the rapid evolution and deployment of computers and communications technology. Second, a growing consumer population is demanding more access to information, more varieties of information and new applications (see Figure 1). Much of this demand is driven by home entertainment. Third, emerging application domains offer opportunities for new applications made possible by the technology and made viable by the potential consumers.

Consumers and application domains are the driving forces behind the technology. In particular, we see a shift from a technological-centric evolution of systems to a solutioncentric one driven by users and applications. Applications in new domains, such as the

\footnotetext{
${ }^{a}$ Centre for Advanced Studies, IBM Canada Laboratory, 844 Don Mills Road, North York, Ontario, Canada M3C 1V7 (e-mail: jslonim@vnet.ibm.com)

${ }^{b}$ Department of Computer Science, Middlesex College Building, The University of Western Ontario, London, Ontario, Canada N6A 5B7 (e-mail: bauer@csd.uwo.ca)
} 
environment, health, and education, become possible because of the new technology. Solutions to problems in these areas require further advances in technology and, we believe, that they require a broader definition of "open systems".

Until now, the concept of an "open system" has been driven by technology experts. If we look at the broader view entailing applications and consumers, open systems become more "real". Moreover, this implies a significant shift in the "perception" of open systems: the concrete reality of applications means that openness is no longer an "if", but a "when". Openness is a key to providing solutions to problems in these domains, and the key aspect of openness is the degree of transparency. The mechanisms involved in how one achieves such transparency, such as CORBA[1] or DCE[2] or ODP[3], become "details". "Proprietary" systems will not disappear; rather, they too will become transparent, especially to the information consumers.

In this paper, we look at the implications of this view on open systems, particularly the challenges that must be addressed in the overall "solution". In other papers $[4,5,6]$ we have looked at some of the technical challenges central to achieving open systems. This paper takes the perspective of the end-user. We look at a particular domain, education, to explore this perspective. The educational domain is of particular interest for two reasons. First, the rapid advances in technology mean that it is very difficult for those in the information-technology field to keep current and maintain and develop new skills. Second, there is an enormous demand for ongoing, life-long learning, in general. Traditional ways of learning, classrooms, universities, etc., are no longer satisfactory. New and emerging technologies will make alternatives possible, and at the same time will raise many issues and challenges for those working on open systems. Some of these will be technological, but many will be economic and societal.

The following section, explores some of the transitions, both technological and societal, already underway. Section examines the requirements and issues arising in life-long learning, including social issues. Section discusses the implications of a broader view of open systems and the challenges facing those developing such systems. The final section provides a brief summary and conclusions.

\section{TRANSITIONS}

The shift toward an information-centric society is being driven by rapid advances in computer and communication technology, increased availability of personal computing systems and software and growth in online, digital information. Trends in some of these areas are illustrated in Figure 2. They are creating changes i.e., transitions [7, 8], in many different aspects of society; global, social and technological.

\subsection{Global transitions}

The emergence of national and global information infrastructures and global computing are changing the way business is done and the ensuing economic issues.

National borders are disappearing. Organizations are becoming increasingly international; different organizations are finding it mutually beneficial to cooperate. Competitiveness is not constrained to or protected by national borders. Advances in communications mean that physical location is no longer a key factor in competitiveness. Work schedules are no longer restricted to 9-5 or east vs. west coast. Information and information technology services are provided as easily by an organization across the street or on the other side of the world. 


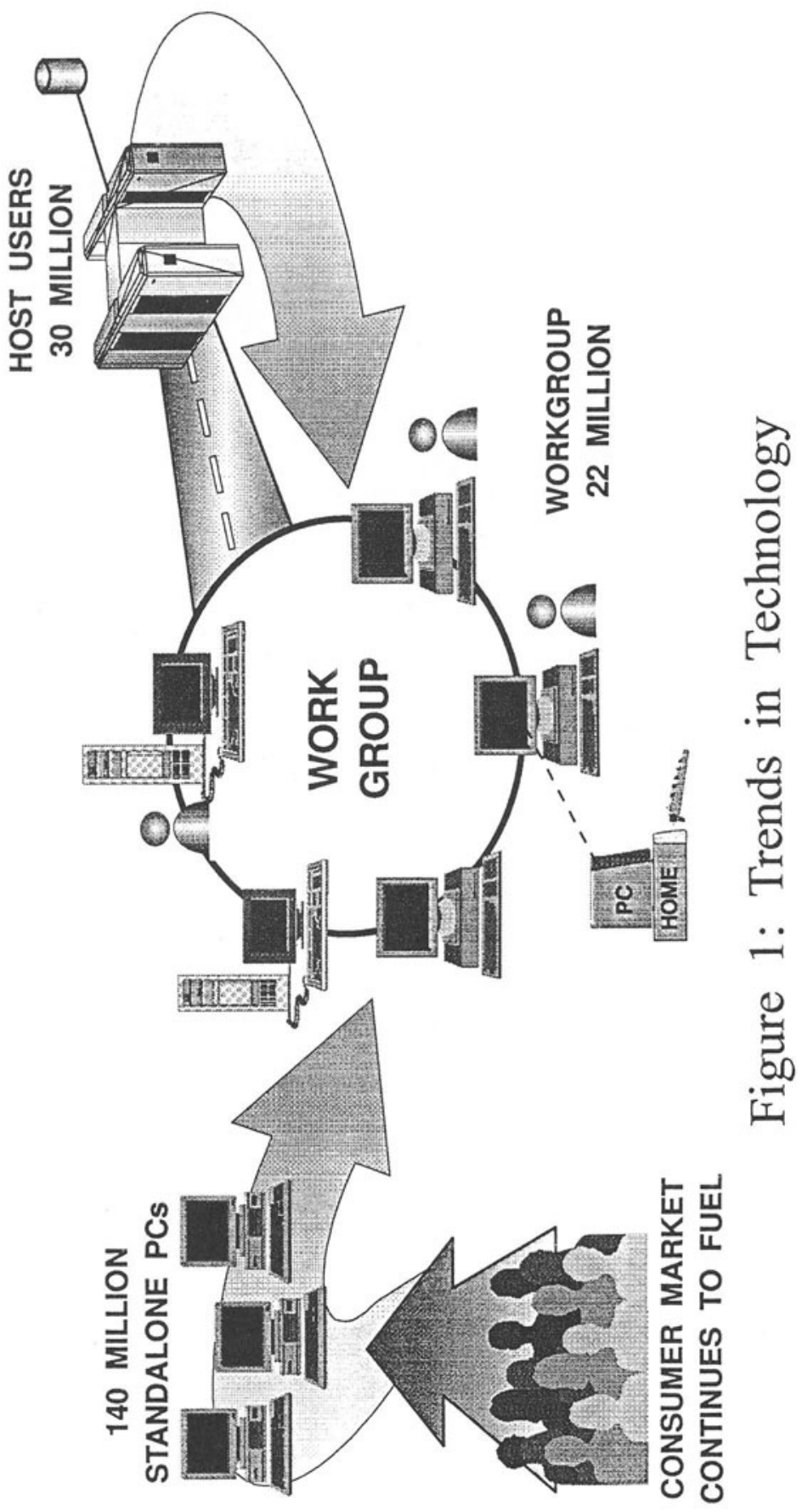


As marketing and sales methods change, new services are emerging. Pizza Hut's recent advertising and accessibility via the internet represent an initial effort to explore these new avenues. In many respects, the services parallel those available via newspapers: advertising, job posting/hunting, current information, etc. The convergence of computer communications, cable television and satellites raises many opportunities in home shopping, advertising and sales services. Access to timely and diverse discussion groups, such as the 60,000 or so available over the internet, means that information exchanges can be dynamic and user-motivated. The provision of these services is not limited to local geographical regions.

As borders become transparent, governments face new challenges. Global competitiveness pushes countries to group together to foster growth, remove restrictions on multinational cooperation, and address regulatory issues. Workers with limited skills are being replaced by knowledge workers. Countries are faced with significant changes in their work forces; appropriate education, will enable them to compete globally. Many of these societal issues are more challenging than the technological issues facing the developers of open systems.

\subsection{Technological transitions}

Coming from technology organizations, most of us are familiar with the transitions within the computer and communication field.

A number of general trends rely on advances in hardware and software. There is a push to provide the means to integrate components, whether they be hardware or software, within an open system framework. We are seeing a shift from centralized computing environments to client-server environments; we believe that this shift will continue toward peer-to-peer computing environments.

The emergence of a consumer population for information and software is forcing the information technology industry to shift from being computer-centric to network-centric and, eventually, to being human-centric. As open networks become reality, the role of the individual workstation will evolve. (For example, see [7]). In the past, there has been a great deal of focus on the individual workstation and its capabilities. With open systems, these workstations become part of a much larger computing environment and are no longer the prime focus. The network will provide access to and will enable use of resources for an individual from any of his or her computers. (An individual may have access to several.) A good analogy is the telephone. The individual telephone has a limited number of well-defined functions and features. A multitude of services can be provided via the telephone if it is connected to the intelligent switches now in operation. The switches provide the additional, value-added services, and they are most critical in communications. As a harbinger for developers of open systems, these switches may involve 20 million lines of code. Competitive advantages go to those who can make changes rapidly without introducing problems.

Further, as consumers make greater use of information technology and place greater demands on what is provided, they will become the driving force behind the technology. This demand will necessitate a further shift toward a human-centric view of information technology. As more users with a limited knowledge of technology gain access to information technology in their jobs, there will be a greater need to focus on user interaction. New devices and modes of communication, for example, voice input and output, will be required. The underlying systems will inevitably change. Interfaces will have to become "standardized", and systems will have to change to comply with these "standards". Again, by way of analogy, consider the telephone; even with some variation, the interface 
is standard.

Interestingly, the shift toward network-centric and human-centric is happening concurrently. As technology drives toward openness, there is a push toward a network-centric view. Simultaneously, as the population of consumers of information technology grows, there is pressure to move toward their view. This additional pressure has motivated our concern for a broader view of "openness".

Some of the trends in hardware have already been described. (See Figure 2). Processors continue to double in speed and capacity every eighteen months. The speed of system-to-system communications is increasing at an incredible rate. Advances in secondary storage technology continues to decrease the cost of a megabyte, whether in memory, on secondary storage, or on optical disks. Hardware components are fast becoming commodities with well-defined and variable characteristics, for example, a processor with a specific clock speed or performance level, a hard disk with a certain access speed or storage capacity. Other device-specific details are often irrelevant to the choice. Other factors are important to the consumer, such as price, availability, and reliability.

The new application domains made plausible through advances in the above technologies and the growth of a consumer population will also require transitions in software technology. These new applications will require domain-specific skills and knowledge and the use of various building blocks, such as objects or processes. It is likely that many of the future applications will be "composed" by domain-specialists from these components or niche applications, such as word processors or spreadsheets. (See Figure 3.) These new applications may also require new languages - a shift from the procedural languages of today to ones which are declarative or functional.

These applications will also demand new forms of data. Structured data will be augmented with unstructured data, such as text, voice, video. New approaches for storing, accessing, and searching such data will be required. No single data model will suffice for all forms of data or applications; multiple models will have to be integrated. Online data sources will grow; terabyte databases will become commonplace and grow to petabyte or exabyte sizes.

We also see a shift from data to information to "knowledge" [9]. Not only will there be growing sources of data, basic digital representations of numbers, characters, audio, video, etc., but there will be an even greater growth in information, that is, a capturing of the relationships among the data. Moreover, the "information" is very likely to grow as it is used by more people and as it is digested and new relationships drawn. Finally, it will become necessary to synthesize this information, that is, to extract "knowledge" from it. "Intelligent agents" may be needed to help users extract knowledge pertinent to their needs.

Mobile computing means that applications and information sources will be accessible from just about anywhere in the world. There is already some movement of jobs from the office to homes. With the prevalence of mobile computing, the "office" will move with the user.

Further, new applications will present much greater challenges in terms of the integration of components. New needs will arise that can only be addressed by a response to the multiple facets of both hardware and software technologies.

Just as there is a shift from mainframe computing environments to distributed ones, there will be a shift from managing centralized computing environments to managing 


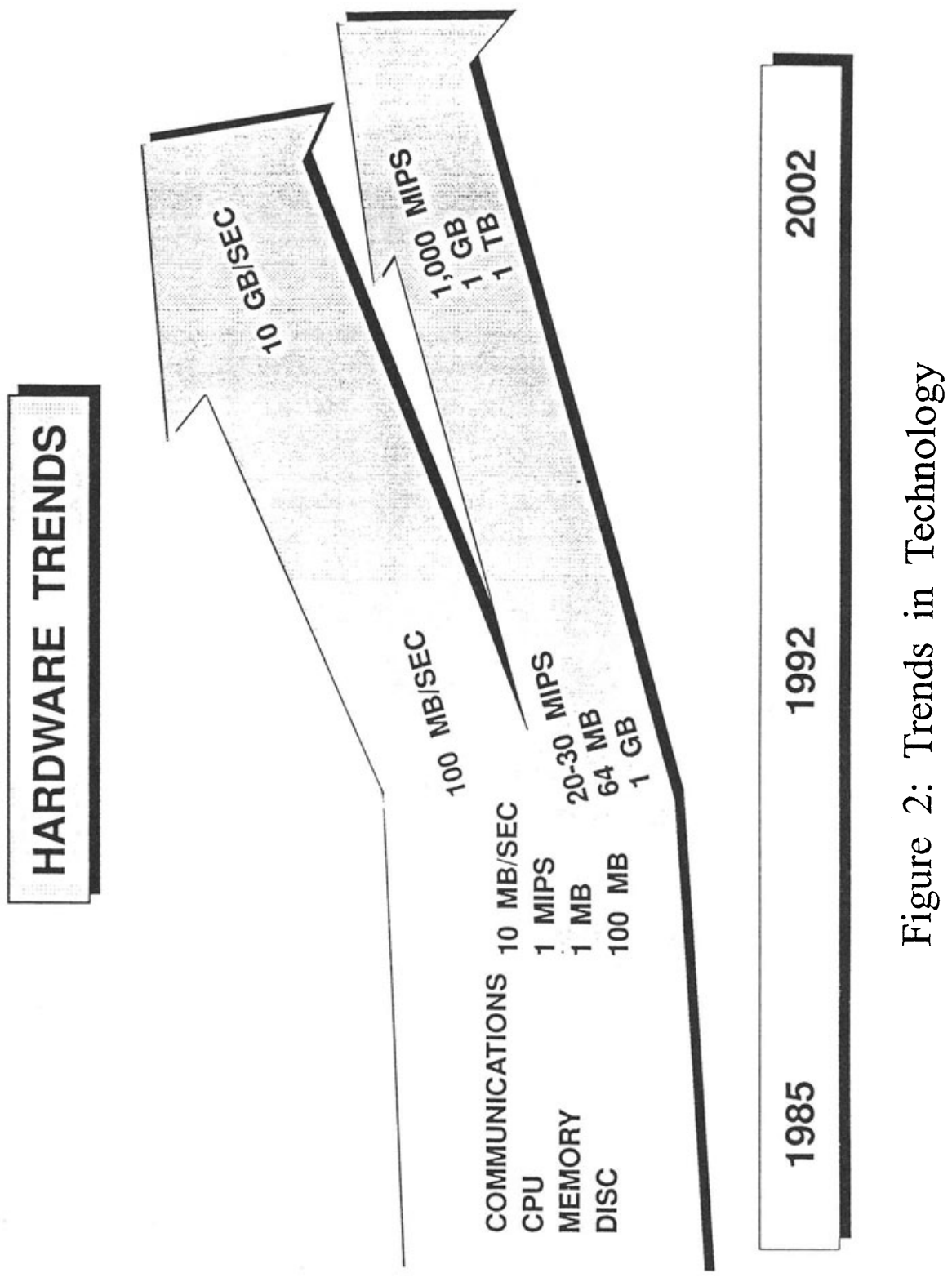




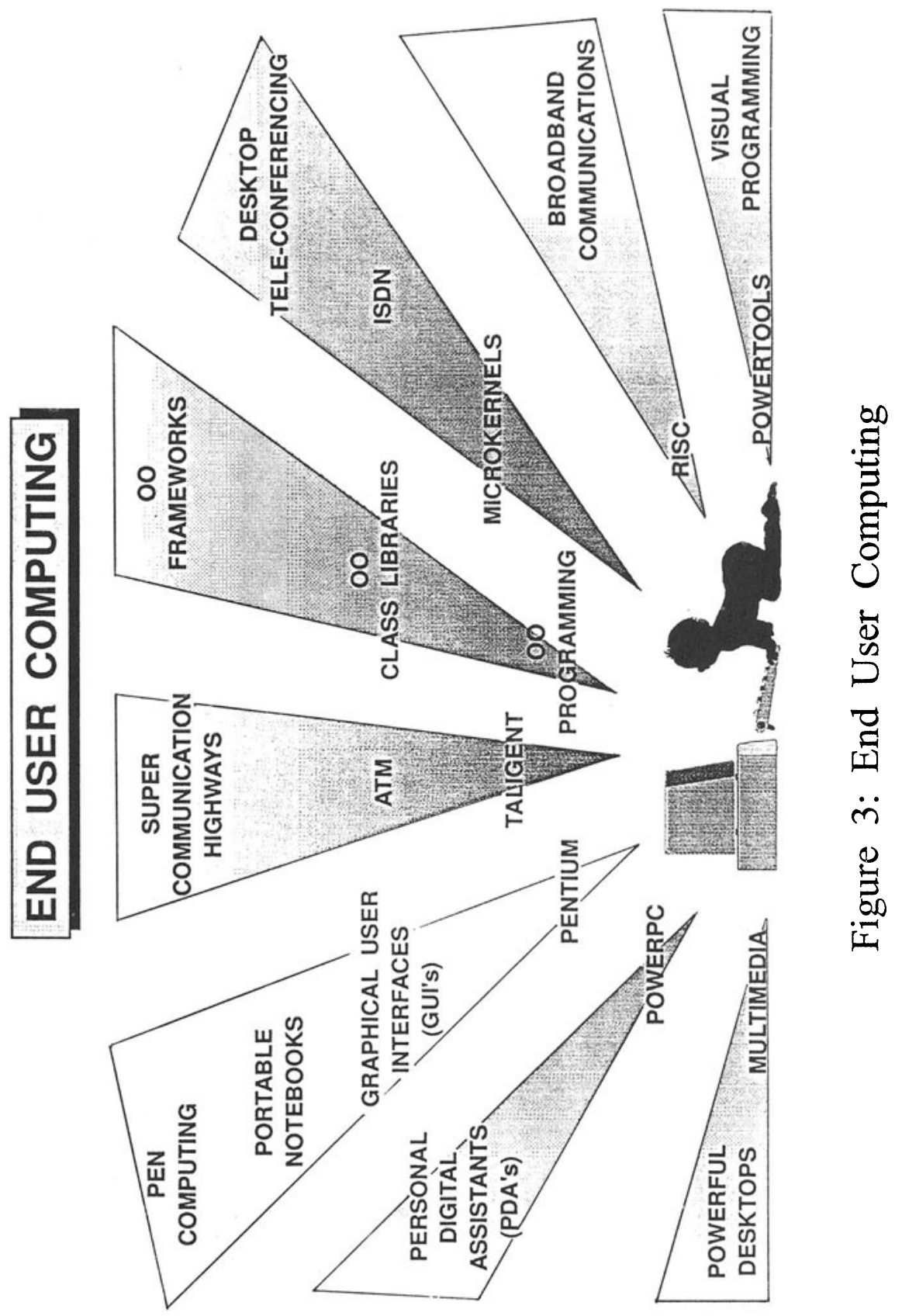


large, distributed computing ones. This shift also presents a number of significant challenges. They include a heterogeneous computing environment, one which is not "static" because the comings and goings of individual workstations and mobile computing devices; the dynamic reallocation of software resources, such as servers; and quality of service requirements for certain applications. All of these will require bandwidth on demand, placing additional complexity on the management of the entire network. Configuration management will become more important as organizations cope with a dynamic, diverse computing environment.

Management will be further complicated as devices and systems are embedded with more "intelligence" of their own. Home computers, televisions, radios, even refrigerators, will be, at least potentially, "manageable". It will be possible to monitor, perhaps even control, millions of devices. Information technology may fundamentally change the home. Delivery of a variety of different types of information, multiple household users of technology, its pervasiveness in many different devices may result in the "home" being simply another extension of the network. As suggested in a recent newspaper article about Bill Gates' home, future homes may be like his and have a wall or walls of one or more screens for communication with others, for entertainment, for learning, etc.

\subsection{Social transitions}

Societal changes are inevitable as we move into an information-based society and the consumers of information technology increase. Information technology will have to become more human-centric; systems and services will have to be designed around users and their needs. There is already a shift from a non-computerized generation to one that is computer literate or at least computer "aware" and an increase in the number of nontechnical users requiring access and use of information technology[10]. A generation of knowledge workers is emerging.

As economies become based on information technology and knowledge-dependent jobs, the common notion of the "work place" will change. Organizations are now experimenting with work at home. As noted, mobile computing will mean that an individual can take the office along. Will this change lead to a generation of individuals unable to interact in social settings?

Distributed work, the pervasiveness of information technology, and many other aspects of an information-based society, threatens the privacy and security of the individual. This concern will have to be addressed through advances in technology and in changes and additions to international regulations and processes.

The growth in users of information technology will have other affects as well. Many jobs requiring minimal skills will be eliminated and replaced with new ones, as yet unknown, requiring knowledge skills. The creation of new applications, for example in the application domains, and new information sources require knowledge workers to acquire new skills on an ongoing basis. Moreover, the individual will have to be more responsible for his or her own education, specifically within the context of an individual job and its needs. Education will have to be timely, accommodate greater numbers of nontechnical users, and have to become personalized. Learning will be life-long.[11, 8]. Individuals will not be able to return to long-term classroom education; education must be continuous. Those providing information technology and open systems as well as those in the education systems must face the challenges of education. Subsequent sections discuss these concerns.

Given the current levels of job-related pressure and stress, how will individuals be able 
to take responsibility for their own ongoing education and spend the time being educated? We cannot continue at the current pace and still expect individuals to remain up-to-date. Education will fail if individuals have no time to relax and to spend time with family and friends. We may have to change our work scenarios so that employment consists of three days of work and two days of self-directed education. Such an employee would still receive remuneration at current levels, but have two days of rest and relaxation in order to maintain effectiveness.

How will those currently unable to afford an education be trained? On one hand, affordable technology may mean that access to educational resources available throughout the network can make education available to many more individuals and at an overall lower cost per student. On the other hand, will such resources be freely available[10]? If there are costs, who will pay? How is continuous education to be funded? Responding to these questions will significantly affect educational systems.

Finally, we are already seeing the beginnings of a merger of information technology with entertainment. What will be the boundaries between entertainment and information, and who will own the content? Will there be boundaries? Should there be? As with the current entertainment industry, how will censorship be handled, especially with the delivery of products across national boundaries, and who will assess the accuracy, etc. of the information?

\section{LIFE-LONG LEARNING}

As information technology will play a greater role in helping to provide ongoing education. The following focuses on ongoing education, commonly called continuing education, and the requirements placed on information technology in general. The implications for open systems, in particular, are discussed in the section. We do not address the totality of the educational environment. Some of the technological advances implied in the following may also be of great use in other aspects of education, such as in K-12.

\subsection{Educational technology requirements}

Advances in information technology, and, in particular, open systems, will make this new education possible[8]. The use of such technology in providing education to the worker does not preclude its use of traditional educational settings. We believe that advances in educational technology for the workplace can augment and expand and, perhaps in some instances, even replace how students are taught in traditional settings[12]. The following points identify some of the requirements of life-long learning:

- the need for timely, accurate information: Information on specific topics or skills must be provided when an individual needs it. Needs may arise during "normal" working hours, but also at other times as individuals take on the responsibility of their own education.

- Need to filter information: The multitude of information sources and enormous amounts of detail mean that a user needs tools to acquire relevant information at the level of detail the user requires. Intelligent agents can help an individual locate sources of information and filter their contents.

- Multiple delivery mechanisms: Similar information might exist in different formats, for example, an audio/video form and a textual one[13]. Different delivery mechanisms will be required to satisfy the needs of different individuals. For example, a 
textual version may be needed by someone wanting to study a topic in great detail, whereas a straightforward audio/video presentation may just provide an overview.

- Geographical transparency: The individual will not be bound to "courses" provided by local institutions, instructors or information sources. Global education resources and expertise will be available. Current efforts at distance education are already moving in this direction.

- Self-paced education: Different individuals will have different needs in the kinds and level of detail and will also learn at different rates. While self-paced instruction may be adequate for updating knowledge, gaining some basic introductory understanding of an area, etc., will it create an expert? How will curricula have to change to accommodate this need? New educational approaches will be required.

- User-oriented delivery: Education must become personalized. Individual needs and preferences must be accommodated. Individuals will study at different times, require different amounts of detail, move at different pace, etc. Software must be provided that can be customized to a user's preferences or that facilitates the user's own selection. Further, physically challenged individuals may require specialized interface devices, software, etc.

- Dynamic and scalable curriculum: In some instances, extended periods of study will be required. An individual may need to study material over weeks, or months, or even longer. Curricula would be required to lead the individual through his or her studies. Such curricula would have to be tailored and customizable based on a user's needs, preferences, capabilities. (See next point.)

- Multidisciplinary: The needs of an individual may require knowledge from several different disciplines. Material from different sources and domains may have to be brought together, perhaps in ways not anticipated by designees.

- Life-long plan for education: As an individual changes jobs and careers, educational needs will change as well. Education will become part of planning a career.

- Need to locate experts: Part of an individual's learning will involve studying bodies of material, but part will also involve consulting with experts. Such consultation will require advanced technology and will require tools to help individuals contact experts.

- Tools for interaction: One advantage of classroom or seminar settings is the ability to learn from other individuals and to debate and discuss ideas. Advances in technology are making online, real-time, multiparty communication and interaction a possibility[14]. Group collaboration is important, such collaboration would also have to be available. The development of interfaces, devices and appropriate curricula will be required to facilitate such interaction.

- Multi-lingual communications: Given the global information community, there may be sources of information or experts anywhere in the world; "courses" may involve individuals from different countries. Simply providing for the exchange of messages will not be enough; agents to help translate or to find experts to translate will be needed.

- Multi-modal interactions: There will also be a need for expanded interaction mechanisms. Keyboards may be adequate for many purposes and users, but others will want to use a routine pen-like device or touch screens to access information and run applications. Better displays and audio will be required for multiple interactions, 
videos, etc. Applications will have to accommodate different combinations of input and output devices depending on the needs and desires of the individual. Interactions may also come via less familiar devices. A user's stereo or television may draw upon digital sources for delivery, diagnosis of device problems, such as a malfunctioning washing machine or automobile, may be handled from remote sources and therefore require connection to the network.

- Convey human emotion: A greater challenge exists in providing the means to convey human emotions. A lecturer's excitement about a particular topic cannot be adequately conveyed via strict textual exchanges; the vehemence of a debate is not effectively conveyed in a news group.

- Entertaining: As the entertainment and information technology industries become more closely aligned, it may be possible to make education fun, exciting as well as rewarding. Expertise from both industries may be needed to effectively deliver educational material to an individual or group of individuals.

- Packaging: Just as curriculum design will need to become more flexible and more individual oriented, there will be a need to combine technologies with sources of information, with services and delivery mechanisms. Information is of little use if the content is limited, if the technology is inadequate to display or deliver the information or if the service is unreliable.

- Ownership, copyrights and plagiarism: There are questions about ownership of online material. In an educational setting, incentives are needed to ensure that the best educators are encouraged to prepare material and interact with students and knowledge workers. How to ensure the intellectual property of on-line information must be addressed. Because digital information makes reproduction of material easy, there must be assurances that an individual's work is his or her own.

- Affordable: Even with great technological advances in hardware and software, the use of information technology for education will not become a reality unless it is affordable. On the other hand, vendors and providers of information must be able to survive in an increasingly competitive world.

- Digital libraries: As more information becomes digitized, we will see a shift from the traditional library to an online one. Intelligent search agents will help locate reference material, perhaps at distant sites. Changes taking place within the digital library framework are discussed in greater detail elsewhere[15].

While these requirements are not necessarily complete, they raise many challenges, especially for those working on open systems. In section, we explore some of these challenges.

\subsection{Socio-economic requirements}

The future learning and education environment represents many challenging technological problems. A number of social (see [16] for a discussion of some) and economic requirements must ultimately be addressed.

First, as suggested above, there are many questions surrounding ownership and copyrights. These will have to be addressed in the broader context of governments and international regulations. 
A second issue arises out of the question of cost. An educational system must be affordable, yet service providers, information providers, integrators, experts, etc., will be unwilling to participate unless they can do so profitably. How will educational "services" be paid for? Should they be free to the individual, funded by governments? Should they be on a pay-per-use basis? How is public education separated from work place education? Should it be?

Security and privacy will continue to be issues, even in the context of education. Providers of information sources will want to ensure that only authorized access to their databases is permitted. In a competitive environment, much can be gleaned by knowing what "research" individuals in one organization are doing. There is a need to ensure that some of the educational activities pursued by an individual are kept personal.

Transparency itself complicates the above issues. Being able to access certain courses or information sources regardless of where they are or who offers them, but there may be cost implications that the user may need to know about. Similarly, not know who is accessing a particular educational provider's material may not be desirable.

Finally, although providing an individually oriented educational environment via technology is an exciting possibility, the overall management of the entire educational infrastructure represents a great challenge. How is access provided for all individuals within a single country? How is online, distance education integrated with local classroom education? How are such services, such as public libraries integrated? How are these, in turn, integrated with digital libraries? How will future teachers and educators themselves be trained (or retrained)? Many of the existing governmental educational structures will have to change to accommodate radical changes in education.

\section{Implications and challenges for open systems}

As we have suggested, there is a need to broaden our view of open systems, where an open system is composed of pieces that can fit together. In the last few years, the view of open systems has been vendor-centric: how to facilitate the cooperation of different vendor systems composed of different hardware, software, communications components. To a great extent, this need has been driven by the rapid advances in hardware and communication technologies. More recently, advances in the development of midware have begun to address system and service interactions.

We believe that there is a need to go further, to consider openness from the perspective of the consumer or from the needs of different application domains. In the following, we discuss some of the implications of these requirements on the notion of openness. Then we briefly discuss the challenges facing the developers of open systems and their constituents.

4.1 Implications The broader view of openness we discuss is primarily motivated by our consideration of education and life-long learning. However, many of the aspects discussed are common to many of the other application domains mentioned in the introduction. Where differences are likely to appear of course, is in the specific individual applications and solutions in those domains.

- User-centric environments: Open systems should be driven by the needs of the users. Users will include traditional students and teachers at all levels of education, as well as knowledge workers seeking to upgrade their skills and expand their knowledge. Users may be individuals at home simply seeking to enhance their quality of life, for example, increasing their gardening skills. 
- Technological transparency: The hardware, communications, and software should be transparent to the end user and should accommodate the user's choice or needs.

- Component independence: Objects, at least those within the domain of relevance to a user, should be independent of underlying systems and platforms; it should be possible to easily move an object from one system to another. More advanced objects may capture data, procedures and models of a user. For example, a "lesson" may have the relevant data, procedures for delivery, testing, etc., as well as model of the user, for example, beginner, expert, preferences, etc., to accommodate delivery and pacing of the lesson itself.

- Representational independence: The user should not know that there are different kinds of data, of different types, stored in different types of database systems. Data should be easily accessible from multiple locations.

- Scalability: Open systems should be scalable to accommodate new systems, applications, devices without visible changes or effects on users. They must also scale to accommodate components of different sizes - systems and data could be potentially unbounded. Building in size limits becomes restrictive, and alternative approaches must be found.

- Peer-to-peer: Open systems will have to evolve from client-server views to peerto-peer; the need for scalability, user-based objects and demands of end users will require the greater flexibility of peer-to-peer.

- Quality of service: The demands of technology throughout the workplace and its role in the knowledge worker's domain will mean that service quality will have to include many aspects of information technology, for example, data access and delivery of information. Open systems will have to be designed to accommodate such service demands.

- Reliability and dependability: Related to quality of service, our view of open systems must be reliable and dependable across multivendor platforms and systems.

- Open, yet proprietary: Openness does not imply that platforms and systems are no longer proprietary. Solutions can still be proprietary, but the interfaces must be open. What is in the box is irrelevant, providing that the interface to the box is open and that it provides what the interface specifies.

- Manageability: As systems become increasingly decentralized and interoperate, management becomes increasingly more complex. Open Systems must be manageable if they are to be successful.

- Charging and costing: Openness implies interoperability and transparency of different vendor systems. For open systems to be successful, it must be possible to recoup costs, even if access and delivery of services cross various geographical boundaries. Certain aspects of charging and costing must be open to enable cooperation, yet there must be sufficient flexibility to permit competition in providing unique, complete or quality information sources and to charge for them.

- Ownership: As information sources become widely accessible, distributed and replicated, and as new generations of applications begin to emerge, such as those in education, questions of ownership and protection become critical. Openness implies accessibility, but, without financial rewards and protection of "information assets", it will not become a reality. 
- Regulatory and business policies: Certain policies, for example, charging, will have to be altered to accommodate open systems. Changes in regulatory policies of governments and specific business policies will be required.

Although many technological problems remain to be addressed before open systems become a reality, transitions already in place suggest that they have become of a question of "when" rather than "if". An expanded view of open systems is required for the understanding and subsequently addressing of technological challenges. Some of these challenges are discussed in the following.

\subsection{Challenges}

Given the requirements of a life-long learning environment, one can identify a number of significant technological challenges. Many of these challenges are also faced in other application domains.

Not surprisingly, the interoperability among systems, services and applications remains a significant challenge. We have seen progress in addressing these issues, but validation of approaches and their scalability remain.

A key to interoperability, since underlying proprietary systems are unlikely to disappear, is the specification and development of interfaces to a variety of components in an open system. What interfaces are required and how are they specified? How do these interfaces evolve as the nature and demands of new applications emerge? How is the consistency of different interfaces ensured, especially in ensuring privacy and security?

Significant challenges exist in ensuring that open systems can provide the appropriate mechanisms for privacy and security. How does one provide openness and interoperability, yet ensure that the openness does not provide unauthorized access to restricted information sources or systems? How can the individual identity of an individual or the actions of an individual be protected? What are appropriate interfaces for ensuring privacy and security concerns between interoperating components?

Open systems will have to accommodate multiple, heterogeneous databases and different types of information. The access, storage and creation of such information must be possible throughout an open system. There may be specialized servers and systems that can provide support for certain types of data, for example, video servers. How is access provided in an open environment? Security and accessibility are critical for vendors supplying such servers.

The multiplicity of information types and sources presents challenges in the delivery infrastructure of open systems. A user at a poor quality device or a mobile device may have to be constrained to a subset of information or even information from a different source. Timely delivery of information across communication channels is only one aspect; quality of service encompasses more.

Open systems will have to encompass peer-to-peer interactions, as argued earlier and elsewhere $[5,6]$. Current work does not address many of the issues in peer-to-peer computing and the composition of components.

Interoperability and the dynamic computing environment likely within an open system mean that the naming and location of resources and systems will be key. It is unlikely that a single naming system will suffice, and even if one is adopted by technology providers, it may be insufficient to support user needs. Directories and name services to accommodate 
multiple naming domains arising in different proprietary platforms, different databases, etc., remain a challenge.

Even with advances in hardware and communication technology, performance will be a primary concern in operational open system environments. Developers need to keep this in mind in designing interfaces for components and in identifying required embedded services. Performance is important in providing quality of service for end-user applications.

Evident in the information technology environment to support life-long learning is the need for agents to operate on behalf of a user. There are challenges in how these agents are developed, their characteristics, behaviour, etc. There are also questions of how they interact, and how security and privacy are ensured, especially since some agents may act on behalf of a single individual, while others may interact with several different individuals.

The need to determine costs means that appropriate accounting mechanisms must be put into place. The open system must provide accounting hooks for multiple vendors, for information providers and for service providers, etc. With access to information from multiple countries and regions, currency conversions, accounting, etc. must be accommodated.

Finally, there is the challenge in creating standards to support open systems. Interfaces between components must be standardized. The focus should not be on standardizing how things are to be done.

\section{SUMMARY AND CONCLUSIONS}

There is major progress in the last five years to build infrastructure, hardware and software, so that new applications can become possible. We need to expand our definition of "openness" to include much more. In particular, we need to move from the technological-driven view of openness to one based on the needs of consumers and applications.

There is a shift in the development environment toward openness integration into the overall networking structure. To address the new domains, developers must address more dynamic issues: configuration management, and systems and application management. In time there must be a move toward total transparency of the technologies themselves. Much more emphasis should be placed on the human-centric aspects of open systems.

As with anything new, requirements and evolving requirements will shift research and development efforts. To find solutions for life-long learning there will be a shift to the digital library; time and place become less important. There will be increased requirements on devices and their connectivity and management. A nontechnical user needs easy-to-use applications. Life-long learning will evolve into a broader discussion of education in general, since many of the issues and requirements are also relevant in public school, high school and universities. Open interfaces are the fuse key to use and acceptance.

At the same time, security, ownership, cost, etc., become magnified in an open environment and must be addressed by researchers. Finally, one should be aware of and prepare for the social issues that open systems raise. 


\section{REFERENCES}

[1] R.M. Soley. Object Management Architecture Guide. Object Management Group, November 1990.

[2] OSF. The OSF Distributed Computing Environment Rationale. Open Software Foundation, Cambridge MA, May 1990.

[3] ITU-TS. Basic Reference Model of Open Distributed Processing Part 1: Overview and Guide to the Use of the Reference Model. ITU-TS Rec X.901, ISO/IEC 10746-1, July 1992.

[4] Michael Bauer, Neil Coburn, Doreen Erickson, Patrick Finnigan, James Hong, Paul Larson, Jan Pachl, Jacob Slonim, David Taylor, and Toby Teorey. A Distributed System Architecture for a Distriubted Application Environment. IBM Systems Journal, 33(3):399-425, September 1994.

[5] Jacob Slonim, Michael Bauer, Patrick Finnigan, Paul Larson, Toby Teorey, Alberto Mendelzon, Richard McBride, Shaula Yemini, and Yechiam Yemini. Distributed Programming Environment: Challenges. Proc. of the International Conference on Open Distributed Processing, pages 379-394, Berlin, Germany, September 1993.

[6] Jacob Slonim, James Hong, Patrick Finnigan, Doreen Erickson, Neil Coburn, and Michael Bauer. Does Midware Provide an Adequate Distributed Application Environment. Proc. of the International Conference on Open Distributed Processing, pages 34-46, Berlin, Germany, September 1993.

[7] Rick Boucher. The Challenge of Transition. EDUCOM Review, 27(5):30-36, September/October 1992.

[8] Information Infrastructure Task Force Committee on Applications and Technology. A transformation of learning: Use of the NII for education and lifelong learning. U.S. Government Printing Office, 1994.

[9] Richard Lucier. Towards Knowledge Management Environment: A Strategic Framework. EDUCOM Review, 27(6):24-31, November/December 1992.

[10] Marilyn Van Bergen. Electronic Citizenship and Social Responsibility. EDUCOM Review, 28(3):45-47, May/June 1993.

[11] Robert Cavalier. Shifting Paradigms in Higher Education and Educational Computing. EDUCOM Review, 27(3):32-35, May/June 1992.

[12] Robert Cavalier. Course processing and the Electronic Agora: Redesigning the Classroom. EDUCOM Review, 27(2):32-37, March/April 1992.

[13] EDUCOM. Multimedia. EDUCOM Review, 27(1):entire issue, January/February 1992.

[14] Elliot Soloway, Mark Guzdial, and Kenneth Hay. Reading and Writing in the 21st Century. EDUCOM Review, 28(1):26-29, January/February 1993.

[15] J.Slonim. Networked Information Systems as Digital Libraries. Technical Report to appear, IBM Academy, January 1995.

[16] EDUCOM. Legal, Social and Ethical Issues: Operating in the '90's. EDUCOM Review, 27(4):entire issue, July/August 1992. 